\title{
Effective properties of particulate nano-composites including Steigmann-Ogden model of material surface
}

\author{
Lidiia Nazarenko $^{1} \cdot$ Henryk Stolarski ${ }^{2} \cdot$ Holm Altenbach ${ }^{1}$
}

Received: 21 October 2020 / Accepted: 30 January 2021 / Published online: 10 March 2021

(c) The Author(s) 2021

\begin{abstract}
The objective of this work is inclusion of the Steigmann-Ogden interface in the Method of Conditional Moments to investigate the influence of surface effects on the effective properties of random particulate composites. The particular focus is centered on accounting for the surface bending stiffness. To this end, the notion of the energy-equivalent inhomogeneity developed for Gurtin-Murdoch interface is generalized to include the surface bending contribution. The crucial aspect of that generalization is identification of the formula defining energy associated with the surface bending. With the help of that formula, the real nano-particle and its surface are replaced by equivalent inhomogeneity with properties incorporating the surface effects. Closed-form expressions for the effective moduli of a composite with a matrix and randomly distributed spherical inhomogeneities are derived. The normalized shear moduli of nanoporous material as a function of void volume fraction is analyzed and evaluated in the context of other theoretical predictions.
\end{abstract}

Keywords Spherical nanoparticles · Composites of stochastic structure $\cdot$ Size-dependent effective properties $\cdot$ Steigmannogden interface model

\section{Introduction}

Interphases between the inhomogeneities and the matrix may have a very pronounced influence on the overall properties of composite materials. At the same time, their inclusion in mechanical (thermal, electric, etc.) analysis of those materials always entails additional complications whose level depends on the complexity of the interphase behavior and on the accuracy, with which that behavior is to be captured analytically. The interphases are typically threedimensional continua but treating them as such is feasible only for simple geometry of the inhomogeneities and for simple loading conditions.

To cover more complex situations, most notably composites involving many interacting inhomogeneities, some

Lidiia Nazarenko

lidiia.nazarenko@ovgu.de

1 Institute of Mechanics, Otto Von Guericke University Magdeburg, Universitätsplatz 2, 39016 Magdeburg, Germany

2 Department of Civil, Environmental and Geo- Engineering, University of Minnesota, 500 Pillsbury Drive S.E., Minneapolis, MN 55455, USA effort has been invested to develop various simplified models of interphases ([1-10] among other). The most practical and popular of them are (arguably) the Gurtin-Murdoch $[11,12]$ model and the spring layer model (e.g., $[1,4,5$, 13-16]. The Gurtin-Murdoch and related models of surface elasticity have been used to study beams, plates, and shells, e.g. Miller and Shenoy [17], Altenbach and Eremeyev [18] among others.

The generalization of Gurtin-Murdoch model was proposed by Steigmann and Ogden [19, 20] who introduced the resistance of the surface to both stretch and bending. It means that the surface energy in the Steigmann-Ogden model includes both the surface strain tensor and the surface curvature tensor. The Steigmann-Ogden model has been used in Chhapadia et al. [21], Mohammadi and Sharma [22] to study bending of nano-sized cantilever beams. In these works the Steigmann-Ogden constants are determined by using combination of atomistic simulations and a simple continuum model.

In Javili et al. [23, 24], dell' Isola and Seppecher [25], dell'Isola et al. [26] it is demonstrated that the higher-gradient theories could entail surface tensors of stresses and couple stresses, as well as other stress resultants. 
Within the Toupin-Mindlin formulation [27-29] of the strain gradient elasticity the mathematical study of static and dynamic boundary value problems with surface stresses described by Steigmann-Ogden model was presented in Eremeyev and Lebedev [30], Eremeev [31]. Similar variational formulation was used in the case of statics of classical elasticity with the Steigmann-Ogden model in Zemlyanova and Mogilevskaya [32]. The effect of curvature-dependent interfacial energy was also studied by Gao et al. [33] for finite deformation. The effective moduli of nanocomposites were analyzed in Gao et al. [34]. The effective properties of the isotropic particulate composites with Steigmann-Ogden interface are derived in Zemlyanova and Mogilevskaya [35]. Cylinder of finite length with Steigmann-Ogden interface was studied in Nazarenko et al. [36].

The main goal of this work is to show that the new concept of energy-equivalent inhomogeneity (EEI), recently presented in [37-39], permits direct evaluation of effective properties of nanomaterials, which includes SteigmannOgden interface model. In addition, we aim at evaluating the effectiveness of the proposed approach by means of numerical examples and comparisons with results obtained using other techniques, also those based on the Lurie solution for sphere [40] in which all governing equations in the inhomogeneity/interphase system are satisfied exactly. Lurie's solution was also used for analysis of the effective behavior of the composites with spherical layered particles in [41, 42].

To illustrate that the presented notion of EEI can be used in combination with any method of evaluating the effective properties of composites, our choice is the method of conditional moments (MCM) rather than the self-consistent approach that is used more commonly.

This article is organized as follows. In the next section the governing equations of the problem of effective properties of random composites with interface is presented. Section 3 deals with the notion of energy-equivalent inhomogeneities. The main contribution of the proposed approach, which is determination of properties of the equivalent spherical inhomogeneity with Steigmann-Ogden interface, is presented in this Section in general terms, with the development quantifying those contributions relegated to Appendices 1 and 2 . Even though the choice of the method used to develop the effective properties of an entire composite is in this work a secondary issue, to make it self-contained in the Sect. 4 a brief description of the MCM and its application to the problems with interphases are provided. The effective properties of particulate composite with Steigmann-Ogden interface are evaluated. Numerical results and comparisons are presented in Sect. 5, while discussion of the approach and conclusions are contained in the last Sect. 6.

\section{Governing equations}

Consider a representative macro-volume $V$ consisting of a matrix with randomly distributed nano-inhomogeneities. Under conditions of uniform loading the macroscopic stress $\overline{\boldsymbol{\sigma}}$ and strain $\overline{\boldsymbol{\varepsilon}}$ are connected by following relations:

$\overline{\boldsymbol{\sigma}}=\mathbf{C}^{*}: \bar{\varepsilon}$,

where $\mathbf{C}^{*}$ is the effective stiffness tensor, and the overbar denotes the operation of the statistical averaging.

For linear elastic materials the problem of finding the effective stiffness tensor requires the solution of the following set of equations.

- Equations of equilibrium:

$\nabla \cdot \boldsymbol{\sigma}(\mathbf{x})=0$,

- Hooke's law:

$$
\boldsymbol{\sigma}(\mathbf{x})=\mathbf{C}(\mathbf{x}): \varepsilon(\mathbf{x}),
$$

- Linear kinematic relation:

$$
\varepsilon(\mathbf{x})=\operatorname{sym}(\nabla \mathbf{u}(\mathbf{x}))
$$

where $\mathbf{x}$ is the position vector of a micro-point.

In Eqs. (2)-(4) $\boldsymbol{\sigma}(\mathbf{x})$ and $\boldsymbol{\varepsilon}(\mathbf{x})$ are the stress and strain tensors in the bulk material (matrix or inhomogeneity), $\mathbf{u}(\mathbf{x})$ is the displacement vector; $\nabla$ is the three dimensional nabla operator; "." identifies the single dot product of two tensors; the fourth-order tensor of elastic parameters $\mathbf{C}(\mathbf{x})$ is a random, statistically homogeneous function of coordinates with a finite scale of correlation and linked to the inclusion and to the matrix properties via

$\mathbf{C}(\mathbf{x})=\mathbf{C}_{1} H(z(\mathbf{x}))+\mathbf{C}_{2} H(-z(\mathbf{x}))$,

where $H$ is the Heaviside function and $\mathbf{C}_{1}$ and $\mathbf{C}_{2}$ denote the values of the tensors of elastic moduli in the inhomogeneities and in the matrix, respectively. The function $z(\mathbf{x})$ is any function satisfying the following requirements:

$\begin{array}{lll}z(\mathbf{x})>0, & \text { if } & \mathbf{x} \in V_{1} \\ z(\mathbf{x})=0, & \text { if } & \mathbf{x} \in S_{I} \\ z(\mathbf{x})<0, & \text { if } & \mathbf{x} \in V_{2},\end{array}$

where $V_{1}$ and $V_{2}$ are the domains of the inhomogeneities and the matrix, respectively and $S_{I}$ is the surface of inhomogeneities.

When the surface (interface) effects are described by the Steigmann-Ogden model [19, 20], Eqs. (2)-(4) need to be supplemented by the equilibrium equations an boundary conditions at the interface $S_{I}$ between the matrix and the nano-inhomogeneities. These relations are derived within 
the Toupin-Mindlin formulation of the strain gradient elasticity in Eremeev [31] and have following form:

displacements continuity on $S_{I}$

$\llbracket \mathbf{u}(\mathbf{x}) \rrbracket_{S_{I}}=\mathbf{0} ;$

Surface equilibrium conditions on $S_{I}$

$$
\begin{aligned}
\llbracket \boldsymbol{\sigma}(\mathbf{x}) \rrbracket_{S_{I}} \cdot \mathbf{n}(\mathbf{x})= & \nabla_{S_{I}} \cdot\left[\boldsymbol{\sigma}_{S}(\mathbf{x})-\left(\nabla_{S_{I}} \cdot \mathbf{M}_{S}(\mathbf{x})\right) \mathbf{n}(\mathbf{x})\right] \\
& -2 H \mathbf{n}(\mathbf{x}) \cdot\left(\nabla_{S_{I}} \cdot \mathbf{M}_{S}(\mathbf{x})\right) \mathbf{n}(\mathbf{x}) .
\end{aligned}
$$

The unit vector $\mathbf{n}$ is normal to $S_{I}$. It is assumed, that at each interface $\mathbf{n}$ points away from the inhomogeneity. The square brackets indicate the jump of the field quantities across the interface, defined as their value on the side towards, which vector $\mathbf{n}$ is pointing minus their value on the side from which it is pointing; $\nabla_{S_{I}}$ is the surface nabla operator; $2 H=\operatorname{tr} \mathbf{B}(\mathbf{x})$ is main curvature; $\mathbf{B}(\mathbf{x})=-\nabla_{S_{I}} \mathbf{n}(\mathbf{x})$ is the curvature tensor; the surface stress tensor $\boldsymbol{\sigma}_{S}[12]$ is defined as

$\boldsymbol{\sigma}_{S}(\mathbf{x})=\tau_{0} \stackrel{2}{\mathbf{I}_{S}}+2\left[\mu_{S}-\tau_{0}\right] \boldsymbol{\varepsilon}_{S}(\mathbf{x})+\left[\lambda_{S}+\tau_{0}\right] \operatorname{tr}\left(\boldsymbol{\varepsilon}_{S}(\mathbf{x})\right) \stackrel{2}{\mathbf{I}_{S}}+\tau_{0} \nabla \mathbf{u}(\mathbf{x})$,

where $\boldsymbol{\varepsilon}_{S}$ is the interface/surface strain tensor, $\mathbf{I}_{S}^{2}$ represents the second-rank identity tensor in the plane tangent to the surface, $\tau_{0}$ is the magnitude of the deformation-independent (residual) surface/interfacial tension (assumed "hydrostatic" and constant in Gurtin-Murdoch model), $\lambda_{S}, \mu_{S}$ are surface Lamé constants, while $\nabla_{S} \mathbf{u}(\mathbf{x})$ denotes the surface gradient of the interface displacement field.

The surface couple stress tensor $\mathbf{M}_{S}$, which describes surface bending $[19,20]$ has following form:

$\mathbf{M}_{S}(\mathbf{X})=2 \mu_{B} \boldsymbol{\kappa}_{S}(\mathbf{X})+\lambda_{B} \operatorname{tr}\left(\boldsymbol{\kappa}_{S}(\mathbf{X})\right) \stackrel{2}{\mathbf{I}_{S}}$.

Here $\lambda_{B}, \mu_{B}$ are additional material parameters describing the bending stiffness of the material surface. The surface strain tensor $\boldsymbol{\varepsilon}_{S}$ and the bending strain measure (tensor of changes of curvature) $\boldsymbol{\kappa}_{S}$

$\boldsymbol{\varepsilon}_{S}(\mathbf{x})=\operatorname{sym}\left(\mathbf{I}_{S}^{2}(\mathbf{X}) \cdot \nabla_{S} \mathbf{u}(\mathbf{x})\right) ;$

$\mathbf{\kappa}_{S}=\operatorname{Sym}\left(\mathbf{I}_{S}^{2}(\mathbf{x}) \nabla_{S} \vartheta(\mathbf{x})\right)$

in which $\vartheta(\mathbf{x})$ is displacement of the end of vector $\mathbf{n}(\mathbf{x})$ due to rotation of the surface

$\vartheta(\mathbf{x})=\nabla_{S_{I}}(\mathbf{n}(\mathbf{x}) \cdot \mathbf{u}(\mathbf{x}))+\mathbf{B}(\mathbf{x}) \cdot \mathbf{u}(\mathbf{x})$.

The system of differential Eqs. (2)-(6), (7) can be transformed to the system of integral equations with the help of
Green's function $\mathbf{G}(\mathbf{x})$, which is defined by the following boundary-value problem

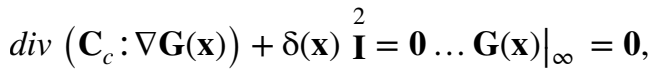

where $\mathbf{C}_{c}$ is the constant tensor describing elasticity of the selected reference medium, $\delta(\mathbf{x})$ denotes the Dirac delta function and $\mathbf{I}$ is the identity tensor of rank two. Then the fluctuations in the displacement field within the entire region $V$ are described by the following formula (see [37, 43]:

$$
\begin{aligned}
\mathbf{u}^{0}(\mathbf{x})= & \int_{V_{y}} \mathbf{G}(\mathbf{x}-\mathbf{y}) \cdot \operatorname{div}\left(\mathbf{C}^{0}(\mathbf{y}): \varepsilon(\mathbf{y})-\boldsymbol{\beta}\right) d V_{y} \\
& -\oint_{S_{y}} \mathbf{G}(\mathbf{x}-\mathbf{y}) \cdot \nabla_{S_{I}} \cdot\left[\sigma_{S}(\mathbf{x})+\left(\nabla_{S_{I}} \cdot \mathbf{M}_{S}(\mathbf{x})\right) \mathbf{n}(\mathbf{x})\right] d s_{y},
\end{aligned}
$$

where $\boldsymbol{\beta}$ is an arbitrary constant.

We consider the macro-volumes and macro-surfaces as infinite (as in physical experiments, they need to be considerably lager than the dimensions of inhomogeneities) and the boundary conditions have the form:

$\left.\mathbf{u}^{0}(\mathbf{x})\right|_{\infty}=\mathbf{0}$.

The linear kinematic relations of Eq. (4) combined with Eq. (15) and with the Gauss theorem leads to the following stochastically non-linear integral equations for the random strain field (see Nazarenko et al. [43] for more details)

$$
\begin{aligned}
\boldsymbol{\varepsilon}(x)= & \overline{\mathbf{\varepsilon}}+\mathbf{K}(\mathbf{x}-\mathbf{y}) *\left[\mathbf{C}^{0}(\mathbf{y}): \boldsymbol{\varepsilon}(\mathbf{y})\right] \\
& -\operatorname{sum}\left\{\nabla_{x} \oint_{S_{I}} \mathbf{G}(\mathbf{x}-\mathbf{y}) \cdot \nabla_{S_{I}} \cdot\left[\sigma_{S}(\mathbf{x})+\left(\nabla_{S_{I}} \cdot \mathbf{M}_{S}(\mathbf{x})\right) \mathbf{n}(\mathbf{x})\right] d S_{Y}\right\} .
\end{aligned}
$$

The operator $\mathbf{K}(\mathbf{x}-\mathbf{y})$ acts according to

$\mathbf{K}(\mathbf{x}-\mathbf{y}) * \boldsymbol{\Psi}(\mathbf{y})=\int_{V} \operatorname{sym}\left(\nabla_{x}\left(\nabla_{x} \mathbf{G}(\mathbf{x}-\mathbf{y})\right)\right):(\boldsymbol{\Psi}(\mathbf{y})-\overline{\boldsymbol{\Psi}}) d V_{y}$,

while $\bar{\varepsilon}$ and $\overline{\boldsymbol{\psi}}$ are mean (expectation) values of $\boldsymbol{\varepsilon}(\mathbf{x})$ and $\boldsymbol{\psi}(\mathbf{y}) \cdot \mathbf{C}^{0}(\mathbf{y})$ in Eq. (17) is the fluctuations in elastic constants and is defined as

$\mathbf{C}^{0}(\mathbf{y})=\mathbf{C}(\mathbf{y})-\mathbf{C}_{c}$.

The issues related to selection of the tensor $\mathbf{C}_{c}$ were discussed in [37, 43-45] and the Reader is referred to these articles.

The way to account for the surface effects described by Eq. (17) is rigorous. However, evaluation of the surface integral containing $\left[\boldsymbol{\sigma}_{S}(\mathbf{x})+\left(\nabla_{S_{I}} \cdot \mathbf{M}_{S}(\mathbf{x})\right) \mathbf{n}(\mathbf{x})\right]$ in the right-hand side of Eq. (17) is a rather complex problem for the case of 
arbitrary loading. In Nazarenko et al. [43], this was done only under some approximating assumptions, valid only for overall volumetric deformations. To bypass that difficulty an approach based on MCM in combination with a notion of the EEI was proposed in [37-39]. In this approach the contribution of the surface integral in Eq. (17) is accounted for by a proper adjustment of the bulk properties of the inhomogeneities, and the material is then analyzed as standard composite, i.e. involving no surfaces possessing their own mechanical characteristics.

According with the above remarks, in the EEI approach Eq. (17) is replaced by the equation for the energy-equivalent system (see [37]

$\varepsilon(\mathbf{x})=\bar{\varepsilon}+\mathrm{K}(\mathbf{x}-\mathbf{y}) *\left[\tilde{\mathbf{C}}^{0}(\mathbf{y}): \varepsilon(\mathbf{y})\right]$,

in which the surface integral of Eq. (17) is incorporated into $\tilde{\mathbf{C}}^{0}(\mathbf{x})$ as a result of changing properties of the inhomogeneities from $\mathbf{C}_{1}$ to $\mathbf{C}_{\text {eq. }}$. Detailed developments leading to $\tilde{\mathbf{C}}^{0}(\mathbf{x})$ are presented in Sect. 3. Tensor $\tilde{\mathbf{C}}^{0}(\mathbf{x})$ is defined as:

$\tilde{\mathbf{C}}^{0}(\mathbf{x})=\tilde{\mathbf{C}}(\mathbf{x})-\mathbf{C}_{C}$ and $\tilde{\mathbf{C}}(\mathbf{x})=\mathbf{C}_{\mathrm{eq}} H(z(\mathbf{x}))+\mathbf{C}_{2} H(-z(\mathbf{x}))$.

Equation (20) is solved in an averaged sense by the MCM to extract the average strains. As described subsequently, this leads to the determination of average stresses and the effective elastic constants.

\section{Energy-equivalent inhomogeneity}

\subsection{The concept of energy-equivalent inhomogeneity}

The idea of the EEI is simple: given that the interfaces in Steigmann-Ogden $[19,20]$ model are coherent (no jump in displacements across the interface), the surface strains of the interface can be related to the strains in the bulk material of the nano-inhomogeneity (or of the matrix). Furthermore, since the two-point approximation of the MCM-typically used in analysis based on that approach-effectively implies that the strains within the nano-inhomogeneities are assumed constant (for details see [43,38]), it is convenient to relate the interface strains to those of the inhomogeneities (not those of the matrix, where the strain field is not assumed constant). Under those conditions it is reasonable to expect that the overall stiffness characteristics of the analyzed nanomaterials should remain essentially the same whether the stiffness provided by the interface is treated independently (as done in [43] or lumped together with the original stiffness of the nano-ihomogeneity. With such modified stiffnesses of all nano-inhomogeneities, combining their original properties and those of their interfaces, the composite may be considered as standard, in which there is no need for an independent inclusion of the surface effects.

The advantage of the approach based on the notion of EEI is that it bypasses all the technical difficulties caused by the presence of independently treated interfaces, encountered for example in Nazarenko et al. [43]. All the formulas for the effective properties of standard random heterogeneous materials (i.e. those not involving interfaces), that can be relatively easily developed using MCM [44, 45], become then directly applicable to nano-materials (i.e. involving interfaces), as long as the properties of inhomogeneities are properly modified to include the surface effects.

To find the modified properties of the EEI it is postulated that, for arbitrary - but homogeneous-deformation, those modified properties render the elastic energy of the EEI that is equal to the sum of the energies of the unmodified inhomogeneity and the energy of its interface. The postulate made here yields:

$\frac{1}{2} V_{1}\left(\bar{\varepsilon}: \mathbf{C}_{\mathrm{eq}}: \bar{\varepsilon}_{1}\right)=\frac{1}{2} V_{1}\left(\bar{\varepsilon}: \mathbf{C}_{1}: \bar{\varepsilon}_{1}\right)+E_{S}$.

Here $\mathbf{C}_{\mathrm{eq}}$ is the stiffness tensor of the modified inhomogeneity to be found, $\mathbf{C}_{1}$ is the stiffness tensor of the original inhomogeneity; $V_{I}$ is the volume of the inhomogeneity and $S_{I}$ is its surface; $\bar{\varepsilon}_{1}$ is the constant strain tensor within the volume of the inhomogeneity; $E_{S}$ is the surface energy.

In the case of Steigmann-Ogden interface Eqs. (7)-(13) the surface energy can be represented as

$E_{S}=U_{T}+U_{B}$,

where $U_{T}$ and $U_{B}$ are the energies related to the surface tension and the surface bending

$U_{T}=\frac{1}{2} \int_{S_{I}} \int_{S_{I}}\left[2 \bar{\mu}_{S} \varepsilon_{S}: \varepsilon_{S}+\bar{\lambda}_{S} \operatorname{tr}\left(\varepsilon_{S}\right)^{2}+\tau_{0} \nabla_{S} \mathbf{u}: \nabla_{S} \mathbf{u}\right] d S ;$

$U_{B}=\frac{1}{2} \int_{S}\left[2 \mu_{b} \mathbf{\kappa}: \mathbf{\kappa}+\lambda_{B} \operatorname{tr}(\boldsymbol{\kappa})^{2}\right] d S$.

In Nazarenko et al. [39], it is shown, that for spherical and isotropic inhomogeneities, and for isotropic surface properties described by Gurtin-Murdoch model of material surface expressed in Eqs. (7)-(9) if $\mathbf{M}_{S}(\mathbf{x})=0$, the stiffness tensor $\mathbf{C}_{\mathrm{eq}}$ of equivalent inhomogeneity is also isotropic and its bulk and shear moduli are defined as

$K_{\text {eq }}=K_{1}+\hat{K}_{T} ; \mu_{\text {eq }}=\mu_{1}+\hat{\mu}_{T}$,

where $K_{1}$ and $\mu_{1}$ are the constants (bulk and shear moduli) of the original inhomogeneity, while $\hat{K}_{T}$ and $\hat{\mu}_{T}$ are (see details in [39] 
$\hat{K}_{T}=2 \frac{\left[2 \bar{\mu}_{S}+2 \bar{\lambda}_{S}+\tau_{0}\right]}{3 a}, \hat{\mu}_{T}=\frac{7 \bar{\mu}_{S}+\bar{\lambda}_{S}+5 \tau_{0}}{5 a}$,

with $\bar{\lambda}_{S}=\lambda_{S}+\tau_{0}, \bar{\mu}_{S}=\mu_{S}-\tau_{0}$ appearing as a result of the surface tension contribution in Eq. (22) and Eq. (8); $a$ is radius of spherical inhomogeneity. If $\mathbf{M}_{S} \neq 0$ in Eqs. (7)-(9), the tensor $\mathbf{C}_{\mathrm{eq}}$ should be also isotropic and its bulk and shear moduli can be defined as

$K_{\text {eq }}=K_{1}+\hat{K}_{T}+\hat{K}_{B} ; \mu_{\text {eq }}=\mu_{1}+\hat{\mu}_{T}+\hat{\mu}_{B}$,

where $\hat{\mu}_{B}$ and $\hat{K}_{B}$ are the additional contribution of the surface bending.

The development, when the term $\mathbf{M}_{S}$ of Eq. (8) is neglected, was presented in [39]. Here the focus of evaluation of the properties of EEI is on the contribution of the surface bending. Use of the complete Eq. (8) in analysis may turn out to be important in some practical applications, where bending of surface should be accounted (e.g. [46]. Inclusion of the complete Eq. (8) within the framework of the EEI is outlined in the next subsection, with some supporting derivations presented in the related "Appendix 1".

\subsection{Contribution of the surface bending to the energy of equivalent inhomogeneity}

\subsubsection{Evaluation of the surface energy related to the bending}

The overarching idea pursued here is the same as that described in the preceding subsection. The only outstanding issue that needs to be addressed is how to determine the surface contribution in Eq. (25) in order to account for the presence of the surface bending in Eq. (8). To this end, the tensor of curvature changes will be evaluated first.

It is assumed, that the strains $\overline{\boldsymbol{\varepsilon}}_{1}$ that an inhomogeneity is subjected to are constant. Under those conditions the displacements in of the surface of that inhomogeneity can be expressed as

$\mathbf{u}\left(\xi^{\Lambda}\right)=\bar{\varepsilon}_{1} \cdot \mathbf{r}\left(\xi^{\Lambda}\right)$,

where $\mathbf{r}\left(\xi^{\Lambda}\right)$ is the position vector of a point on that surface which is locally parameterized by $\xi^{\Lambda}, \Lambda \in\{1,2\}$. Consequently, (cf. [47]

$$
\begin{aligned}
\nabla_{S} \mathbf{u} & =\left(\varepsilon_{1} \cdot \mathbf{r}\right)_{, \Delta} \otimes \mathbf{G}^{\Delta}=\bar{\varepsilon}_{1} \cdot\left(\mathbf{r}_{, \Delta} \otimes \mathbf{G}^{\Delta}\right) \\
& =\bar{\varepsilon}_{1} \cdot\left(\mathbf{G}_{\Delta} \otimes \mathbf{G}^{\Delta}\right)=\bar{\varepsilon}_{1} \cdot{ }_{S} \mathbf{I} .
\end{aligned}
$$

Here $\mathbf{G}_{\Delta}=\mathbf{r},_{\Delta}$ are the vectors of the natural basis associated with the parametrization $\xi^{\Delta}$ (tangent to the surface) and $\mathbf{G}^{\Delta}$ are the vectors of the dual, or reciprocal, basis also
Table 1 Curvature tensors for sphere of radius $a$

\begin{tabular}{llll}
\hline$\Lambda$ & $\Delta$ & $B_{\Lambda \Delta}$ & $B_{\Lambda}^{\Delta}$ \\
\hline 1 & 1 & $-a \sin ^{2} \theta$ & $-1 / a$ \\
1 & 2 & 0 & 0 \\
2 & 2 & $-a$ & $-1 / a$ \\
2 & 1 & 0 & 0 \\
\hline
\end{tabular}

tangent to the surface) satisfying the condition $\mathbf{G}_{\Delta} \cdot \mathbf{G}^{\Lambda}=\delta_{\Delta}^{\Lambda}$ with $\delta_{\Delta}^{\Lambda}$ being the "Kronecker delta".

The tensor of curvature changes is determined as

$\boldsymbol{\kappa}=\operatorname{sym}\left(\stackrel{2}{\mathbf{I}_{S}} \cdot \nabla_{S} \vartheta\right)$,

with

$\vartheta=\boldsymbol{\omega}_{N} \cdot \mathbf{n}$,

where

$\boldsymbol{\omega}_{N}=-\left(\mathbf{n} \cdot \nabla_{S} \mathbf{u}\right) \otimes \mathbf{n}$.

Considering Eq. (30) $\vartheta$ can be defined as

$\vartheta=-\mathbf{n} \cdot \overline{\boldsymbol{\varepsilon}}_{1} \cdot{\stackrel{2}{\mathbf{I}_{S}}}^{=}-\overline{\boldsymbol{\varepsilon}}_{1}: \mathbf{n} \otimes \stackrel{2}{\mathbf{I}_{S}}$,

which gives

$\nabla_{S} \vartheta=-\bar{\varepsilon}_{1}: \nabla_{S}\left(\mathbf{n} \otimes \mathbf{I}_{S}^{2}\right) ;$

$\boldsymbol{\kappa}=-\operatorname{sym}\left[\mathbf{I}_{S}^{2} \cdot\left(\overline{\boldsymbol{\varepsilon}}_{1}: \nabla_{S}\left(\mathbf{n} \otimes{\stackrel{2}{\mathbf{I}_{S}}}^{2}\right)\right)\right]$

The above formula indicate that $\bar{\varepsilon}_{1}$ contracted with the first 2 vectors of $\nabla_{S}\left(\mathbf{n} \otimes \stackrel{2}{\mathbf{I}}_{S}\right)$ and $\stackrel{2}{\mathbf{I}}_{S}$ operate on the third vector of dyadic product in $\nabla_{S}\left(\mathbf{n} \otimes \mathbf{I}_{S}^{2}\right)$. This means that multiplication by $\mathbf{I}_{S}$ eliminate the $\mathbf{n} \otimes \mathbf{G}_{\Lambda} \otimes \mathbf{n} \otimes \mathbf{G}^{\Delta}$ $\left(\mathbf{G}_{\Lambda} \perp \mathbf{n}\right)$ and the remaining two parts are unchanged. So

$$
\begin{aligned}
\boldsymbol{\kappa}= & -\operatorname{sym}\left[\mathbf{I}_{S} \cdot\left(\nabla_{S}\left(\mathbf{n} \otimes \mathbf{I}_{S}\right)\right)^{T}: \overline{\boldsymbol{\varepsilon}}_{1}\right] \\
\boldsymbol{\kappa}= & -\operatorname{sym}\left[\mathbf{I}_{S} \cdot\left(\nabla_{S}\left(\mathbf{n} \otimes \mathbf{I}_{S}\right)\right)^{T}: \overline{\boldsymbol{\varepsilon}}_{1}\right] \\
& =-\operatorname{sym}\left[\left(-B_{\Delta}^{\Pi} \mathbf{G}_{\Pi} \otimes \mathbf{I}_{S} \otimes \mathbf{G}^{\Delta}+B_{\Lambda \Delta} \mathbf{n} \otimes \mathbf{n} \otimes \mathbf{G}^{\Lambda} \otimes \mathbf{G}^{\Delta}\right): \overline{\boldsymbol{\varepsilon}}_{1}\right] .
\end{aligned}
$$

Evaluation of the components of the tensors $B_{\Lambda \Delta}$ and $B_{\Delta}^{\Pi}$ is illustrated in "Appendix 1", where curvature tensors for spherical inhomogeneity of radius $a$ are given in Table 1 .

Considering values $B_{\Lambda \Delta}$ and $B_{\Lambda}^{\Delta}$ from Table 
$\boldsymbol{\kappa}=-\operatorname{sym}\left[\frac{1}{a}\left(\mathbf{G}^{\Lambda} \otimes \mathbf{G}^{1} \otimes \mathbf{G}_{1} \otimes \mathbf{G}_{\Lambda}+\mathbf{G}^{\Lambda} \otimes \mathbf{G}^{2} \otimes \mathbf{G}_{2} \otimes \mathbf{G}_{\Lambda}\right.\right.$

$\left.\left.-\mathbf{G}^{1} \otimes \mathbf{G}_{1} \otimes \mathbf{n} \otimes \mathbf{n}-\mathbf{G}^{2} \otimes \mathbf{G}_{2} \otimes \mathbf{n} \otimes \mathbf{n}\right): \bar{\varepsilon}_{1}\right]$.

Given that for $\mathbf{G}_{\Delta} \cdot \mathbf{G}_{\Lambda}=0$ if $\Delta \neq \Lambda$, the curvilinear coordinates $\xi^{\Lambda}$ introduced to parameterize the surface of the inhomogeneities are orthogonal, the unit vectors $\overline{\mathbf{G}}_{\Delta}=\frac{\mathbf{G}_{\Delta}}{\left|\mathbf{G}_{\Delta}\right|}=\overline{\mathbf{G}}^{\Delta}=\frac{\mathbf{G}^{\Delta}}{\left|\mathbf{G}^{\Delta}\right|}$ can be substituted for $\mathbf{G}_{\Delta}$ and $\mathbf{G}^{\Delta}$ in the above equation and it can be rewritten in the form: energy related to the surface bending. In the next section, the working formula for the properties of the equivalent inhomogeneity is presented.

\subsubsection{Constitutive tensor of the energy-equivalent inhomogeneity}

Considering Eq. (40) last two terms of Eq. (41) are given as

$$
\begin{aligned}
\boldsymbol{\kappa}= & -\operatorname{sym}\left[\frac { 1 } { a } \left(\left[\left(\overline{\mathbf{G}}_{1} \otimes \overline{\mathbf{G}}_{1} \otimes \overline{\mathbf{G}}_{1} \otimes \overline{\mathbf{G}}_{1}+\overline{\mathbf{G}}_{2} \otimes \overline{\mathbf{G}}_{1} \otimes \overline{\mathbf{G}}_{1} \otimes \overline{\mathbf{G}}_{2}+\overline{\mathbf{G}}_{1} \otimes \overline{\mathbf{G}}_{2} \otimes \overline{\mathbf{G}}_{2} \otimes \overline{\mathbf{G}}_{1}+\right.\right.\right.\right. \\
& \left.\left.+\overline{\mathbf{G}}_{2} \otimes \overline{\mathbf{G}}_{2} \otimes \overline{\mathbf{G}}_{2} \otimes \overline{\mathbf{G}}_{2}-\overline{\mathbf{G}}_{1} \otimes \overline{\mathbf{G}}_{1} \otimes \mathbf{n} \otimes \mathbf{n}-\overline{\mathbf{G}}_{2} \otimes \overline{\mathbf{G}}_{2} \otimes \mathbf{n} \otimes \mathbf{n}\right): \overline{\boldsymbol{\varepsilon}}_{1}\right] .
\end{aligned}
$$

Accounting for symmetry of $\bar{\varepsilon}_{1}$ tensor of curvature changes can be defined as

$$
\begin{aligned}
\boldsymbol{\kappa}=- & \frac{1}{a}\left[\left(\overline{\mathbf{G}}_{1} \otimes \overline{\mathbf{G}}_{1} \otimes \overline{\mathbf{G}}_{1} \otimes \overline{\mathbf{G}}_{1}+\left(\overline{\mathbf{G}}_{2} \otimes \overline{\mathbf{G}}_{1}+\overline{\mathbf{G}}_{1} \otimes \overline{\mathbf{G}}_{2}\right)\right.\right. \\
\otimes & \overline{\mathbf{G}}_{1} \otimes \overline{\mathbf{G}}_{2}+\overline{\mathbf{G}}_{2} \otimes \overline{\mathbf{G}}_{2} \otimes \overline{\mathbf{G}}_{2} \otimes \overline{\mathbf{G}}_{2} \\
& \left.\left.-\overline{\mathbf{G}}_{1} \otimes \overline{\mathbf{G}}_{1} \otimes \mathbf{n} \otimes \mathbf{n}-\overline{\mathbf{G}}_{2} \otimes \overline{\mathbf{G}}_{2} \otimes \mathbf{n} \otimes \mathbf{n}\right): \overline{\boldsymbol{\varepsilon}}_{1}\right] .
\end{aligned}
$$

$\boldsymbol{\kappa}: \boldsymbol{\kappa}=\frac{1}{a^{2}} \overline{\boldsymbol{\varepsilon}}_{1}:\left[\overline{\mathbf{G}}_{1} \otimes \overline{\mathbf{G}}_{1} \otimes \overline{\mathbf{G}}_{1} \otimes \overline{\mathbf{G}}_{1}+\overline{\mathbf{G}}_{2} \otimes \overline{\mathbf{G}}_{2}\right.$

$$
\begin{aligned}
& \otimes \overline{\mathbf{G}}_{2} \otimes \overline{\mathbf{G}}_{2}+2 \overline{\mathbf{G}}_{1} \otimes \overline{\mathbf{G}}_{2} \otimes \overline{\mathbf{G}}_{1} \otimes \overline{\mathbf{G}}_{2} \\
& -\overline{\mathbf{G}}_{1} \otimes \overline{\mathbf{G}}_{1} \otimes \mathbf{n} \otimes \mathbf{n}-\overline{\mathbf{G}}_{2} \\
& \otimes \overline{\mathbf{G}}_{2} \otimes \mathbf{n} \otimes \mathbf{n}-\mathbf{n} \otimes \mathbf{n} \otimes \overline{\mathbf{G}}_{1} \otimes \overline{\mathbf{G}}_{1}-\mathbf{n} \\
& \left.\otimes \mathbf{n} \otimes \overline{\mathbf{G}}_{2} \otimes \overline{\mathbf{G}}_{2}+2 \mathbf{n} \otimes \mathbf{n} \otimes \mathbf{n} \otimes \mathbf{n}\right]: \overline{\boldsymbol{\varepsilon}}_{1} ;
\end{aligned}
$$

$$
\begin{aligned}
\operatorname{tr}(\mathbf{\kappa})^{2}= & \frac{1}{a^{2}} \overline{\boldsymbol{\varepsilon}}_{1}:\left[\overline{\mathbf{G}}_{1} \otimes \overline{\mathbf{G}}_{1} \otimes \overline{\mathbf{G}}_{1} \otimes \overline{\mathbf{G}}_{1} \otimes \overline{\mathbf{G}}_{1}+\overline{\mathbf{G}}_{2} \otimes \overline{\mathbf{G}}_{2} \otimes \overline{\mathbf{G}}_{2} \otimes \overline{\mathbf{G}}_{2}+\overline{\mathbf{G}}_{1} \otimes \overline{\mathbf{G}}_{1} \otimes \overline{\mathbf{G}}_{2} \otimes \overline{\mathbf{G}}_{2} \otimes \overline{\mathbf{G}}_{2} \otimes \overline{\mathbf{G}}_{2} \otimes \overline{\mathbf{G}}_{1} \otimes \overline{\mathbf{G}}_{1}\right. \\
& \left.-2 \overline{\mathbf{G}}_{1} \otimes \overline{\mathbf{G}}_{1} \otimes \mathbf{n} \otimes \mathbf{n}-2 \overline{\mathbf{G}}_{2} \otimes \overline{\mathbf{G}}_{2} \mathbf{n} \otimes \mathbf{n}-2 \mathbf{n} \otimes \mathbf{n} \otimes \overline{\mathbf{G}}_{1} \otimes \overline{\mathbf{G}}_{1}-2 \mathbf{n} \otimes \mathbf{n} \otimes \overline{\mathbf{G}}_{2} \otimes \overline{\mathbf{G}}_{2}+4 \mathbf{n} \otimes \mathbf{n} \otimes \mathbf{n} \otimes \mathbf{n}\right]: \overline{\boldsymbol{\varepsilon}}_{1}
\end{aligned}
$$

Then the surface strain energy in the case of SteigmannOgden model of interface is defined as (see for details [31, 32])

$$
\begin{aligned}
& E_{S}=\frac{1}{2} \oint_{S_{I}}\left[2 \bar{\mu}_{S} \varepsilon_{S}: \varepsilon_{S}+\bar{\lambda}_{S} t r\left(\varepsilon_{S}\right)^{2}\right. \\
& \left.+\tau_{0} \nabla_{S} \mathbf{u}: \nabla_{S} \mathbf{u}+2 \mu_{B} \mathbf{\kappa}: \mathbf{\kappa}+\lambda_{B}(\operatorname{tr} \mathbf{\kappa})^{2}\right] d S \\
& U_{\mu_{B}}=\frac{1}{2 a^{2}} \oint_{S_{I}}\left[\bar{\varepsilon}_{1}:\left(\overline{\mathbf{G}}_{1} \otimes \overline{\mathbf{G}}_{1} \otimes \overline{\mathbf{G}}_{1} \otimes \overline{\mathbf{G}}_{1}+\overline{\mathbf{G}}_{2} \otimes \overline{\mathbf{G}}_{2} \otimes \overline{\mathbf{G}}_{2} \otimes \overline{\mathbf{G}}_{2}\right.\right. \\
& +2 \overline{\mathbf{G}}_{1} \otimes \overline{\mathbf{G}}_{2} \otimes \overline{\mathbf{G}}_{1} \otimes \overline{\mathbf{G}}_{2}-\overline{\mathbf{G}}_{1} \otimes \overline{\mathbf{G}}_{1} \otimes \mathbf{n} \otimes \mathbf{n} \\
& \left.\left.-\overline{\mathbf{G}}_{2} \otimes \overline{\mathbf{G}}_{2} \otimes \mathbf{n} \otimes \mathbf{n}-\mathbf{n} \otimes \mathbf{n} \otimes \overline{\mathbf{G}}_{1} \otimes \overline{\mathbf{G}}_{1}-\mathbf{n} \otimes \mathbf{n} \otimes \overline{\mathbf{G}}_{2} \otimes \overline{\mathbf{G}}_{2}+2 \mathbf{n} \otimes \mathbf{n} \otimes \mathbf{n} \otimes \mathbf{n}\right): \overline{\boldsymbol{\varepsilon}}_{1}\right] d S
\end{aligned}
$$

The surface energy of Eq. (41) is a sum of the surface tension and the surface bending Eqs. (23)-(25) and we focus only on the latter

$U_{B}=\frac{1}{2} \oint_{S}\left[2 \mu_{B} \mathbf{\kappa}: \mathbf{\kappa}+\lambda_{B} \operatorname{tr}(\mathbf{\kappa})^{2}\right] d S=U_{\mu_{B}}+U_{\lambda_{B}}$,

where $U_{\mu_{B}}=\frac{1}{2} \oint_{S}\left[2 \mu_{B} \mathbf{\kappa}: \mathbf{\kappa}\right] d S$ is defined as
The first three terms of the above integrand are identical with the surface strain energy given by Gurtin-Murdoch model and properties of equivalent inhomogeneities related to these terms are determined in Nazarenko et al. [37-39]. The last two terms of Eq. (41) represent the surface strain

$$
\text { and } U_{\lambda_{B}}=\oint_{S}\left[\lambda_{B} \operatorname{tr}(\mathbf{\kappa})^{2}\right] d S \text { is }
$$




$$
\begin{aligned}
U_{\lambda_{B}}= & \frac{1}{2 a^{2}} \oint_{S_{I}}\left[\bar{\varepsilon}_{1}:\left(\overline{\mathbf{G}}_{1} \otimes \overline{\mathbf{G}}_{1} \otimes \overline{\mathbf{G}}_{1} \otimes \overline{\mathbf{G}}_{1}+\overline{\mathbf{G}}_{2} \otimes \overline{\mathbf{G}}_{2} \otimes \overline{\mathbf{G}}_{2} \otimes \overline{\mathbf{G}}_{2}+\overline{\mathbf{G}}_{1} \otimes \overline{\mathbf{G}}_{1} \otimes \overline{\mathbf{G}}_{2} \otimes \overline{\mathbf{G}}_{2}\right.\right. \\
& +\overline{\mathbf{G}}_{2} \otimes \overline{\mathbf{G}}_{2} \otimes \overline{\mathbf{G}}_{1} \otimes \overline{\mathbf{G}}_{1}-2 \overline{\mathbf{G}}_{1} \otimes \overline{\mathbf{G}}_{1} \otimes \mathbf{n} \otimes \mathbf{n} \\
& \left.\left.-2 \overline{\mathbf{G}}_{2} \otimes \overline{\mathbf{G}}_{2} \otimes \mathbf{n} \otimes \mathbf{n}-2 \mathbf{n} \otimes \mathbf{n} \otimes \overline{\mathbf{G}}_{1} \otimes \overline{\mathbf{G}}_{1}-2 \mathbf{n} \otimes \mathbf{n} \otimes \overline{\mathbf{G}}_{2} \otimes \overline{\mathbf{G}}_{2}+4 \mathbf{n} \otimes \mathbf{n} \otimes \mathbf{n} \otimes \mathbf{n}\right): \bar{\varepsilon}_{1}\right] d S .
\end{aligned}
$$

These last formulas Eqs. (45), (46) can be put in Eq. (44) and the following form of the surface energy yields

$\mathrm{E}_{S}=U_{T}+U_{\mu_{B}}+U_{\lambda_{B}}=\bar{\varepsilon}_{1}:\left(\mathbf{K}_{T}+\mathbf{K}_{\mu_{B}}+\mathbf{K}_{\lambda_{B}}\right): \bar{\varepsilon}_{1}$,

where

$U_{T}=\bar{\varepsilon}_{1}: \mathbf{K}_{T}: \bar{\varepsilon}_{1} ; U_{\mu_{B}}=\bar{\varepsilon}_{1}: \mathbf{K}_{\mu_{B}}: \bar{\varepsilon}_{1} ; U_{\lambda_{B}}=\bar{\varepsilon}_{1}: \mathbf{K}_{\lambda_{B}}: \bar{\varepsilon}_{1}$.

The last result for $E_{S}$ and the energy equivalence expressed by Eq. (22) leads to the following formula for the effective moduli of equivalent inhomogeneity:

$\mathbf{C}_{\text {eq }}=\mathbf{C}_{1}+\frac{1}{V_{I}}\left(\mathbf{K}_{T}+\mathbf{K}_{\mu_{B}}+\mathbf{K}_{\lambda_{B}}\right)$

Then the problem of properties of equivalent inhomogeneities is reduced to evaluation of the components of the above tensors $\mathbf{K}_{\mu_{B}}$ and $\mathbf{K}_{\lambda_{B}}$, which is illustrated in "Appendix 2" Eqs. (98), (99). Contribution of the surface bending in this case is

$\hat{K}_{B}=0, \hat{\mu}_{B}=\frac{15 \mu_{B}+9 \lambda_{B}}{5 a^{3}}$,

where $\lambda_{B}, \mu_{B}$ are additional material parameters describing the bending stiffness of the material surface Eq. (9). In the presence of $\mathbf{M}_{S}$ in Eqs. (7)-(13), the tensor $\mathbf{C}_{\mathrm{eq}}$ is also isotropic and its constants can be defined as

$K_{\mathrm{eq}}=K_{1}+\hat{K}_{T} ; \mu_{\mathrm{eq}}=\mu_{1}+\hat{\mu}_{T}+\hat{\mu}_{B}$,

where $\hat{\mu}_{T}$ and $\hat{K}_{T}$ are defined in Eq. (26).

\section{Effective properties}

\subsection{Basic features of MCM}

Details of the evaluation of the effective stiffness tensor for composites with randomly distributed spherical particles are presented in Nazarenko et al. [38, 44]. Applying the methodology described in those papers, the following expression for effective moduli of composite with perfect interphase is obtained:

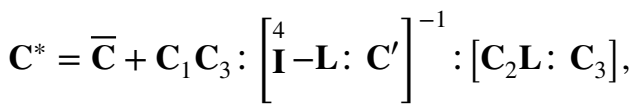

where $\overline{\mathbf{C}}, \mathbf{C}_{3}$ and $\mathbf{C}^{\prime}$ are

$\overline{\mathbf{C}}=\sum_{k=1}^{2} c_{k} \mathbf{C}_{k}, \mathbf{C}_{3}=\mathbf{C}_{1}-\mathbf{C}_{2}, \mathbf{C}^{\prime}=c_{1} \mathbf{C}_{2}+c_{2} \mathbf{C}_{1}-\mathbf{C}_{c}$,

while $\mathbf{C}_{c}$ is the constitutive tensor for the "reference medium" whose selection is specified subsequently. It is shown Nazarenko et al. [45] that the tensor $\mathbf{L}$ in Eq. (52) coincides with the negative classical Hill tensor $\mathbf{P}$ $\left(\mathbf{P}=\mathbf{S}: \mathbf{C}_{2}^{-1}\right.$, with $\mathbf{S}$ being the Eshelby tensor; c.f. [48]. The difference is that the Hill tensor $\mathbf{P}$ is defined using properties of the matrix material whereas tensor $\mathbf{L}$ is related to the stiffness of the reference medium $\mathbf{C}_{c}$.

$\mathbf{L}=2 b \stackrel{4}{\mathbf{I}}+a^{\mathbf{I}} \otimes \stackrel{2}{\mathbf{I}}$,

with

$a=\frac{\lambda_{c}+\mu_{c}}{15 \mu_{c}\left[\lambda_{c}+2 \mu_{c}\right]}, b=-\frac{3 \lambda_{c}+8 \mu_{c}}{30 \mu_{c}\left[\lambda_{c}+2 \mu_{c}\right]}$,

and with $\lambda_{c}, \mu_{c}$ being the Lamé constants of the reference medium defined according to the rule Nazarenko et al. [37]

$\lambda_{c}=\left\{\begin{array}{ll}c_{1} \lambda_{1}+c_{2} \lambda_{2}, & \text { if } \lambda_{1} \leq \lambda_{2} \\ {\left[c_{1}\left(\lambda_{1}\right)^{-1}+c_{2}\left(\lambda_{2}\right)^{-1}\right]^{-1},} & \text { if } \lambda_{1} \geq \lambda_{2}\end{array}\right.$,

$\mu_{c}= \begin{cases}c_{1} \mu_{1}+c_{2} \mu_{2}, & \text { if } \mu_{1} \leq \mu_{2} \\ {\left[c_{1}\left(\mu_{1}\right)^{-1}+c_{2}\left(\mu_{2}\right)^{-1}\right]^{-1},} & \text { if } \mu_{1} \geq \mu_{2}\end{cases}$

\subsection{Application of MCM to problems with interfaces}

The effective properties of the composite with Steigmann-Ogden model of interfaces can be obtained by MCM from Eqs. (52)-(57), valid for perfect interfaces, if the properties of the spherical particles $\left(\mathbf{C}_{1}\right)$ is replaced with those of equivalent inhomogeneity $\left(\mathbf{C}_{\mathrm{eq}}\right)$ and, evaluated in Sect. 3.2.3. This leads to the following expression 


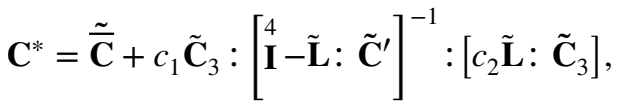

where $\tilde{\overline{\mathbf{C}}}, \tilde{\mathbf{C}}_{3}, \tilde{\mathbf{L}}$, and $\tilde{\mathbf{C}}^{\prime}$ are determined in accordance with Eqs. (53)-(55) if $\mathbf{C}_{1}$ is replaced by $\mathbf{C}_{\text {eq: }}$.

The scalar expression for the effective bulk and shear moduli of the composite can be obtained from a tensorial formula (52), cf. [37, 38]:

$K^{*}=c_{1} \mathrm{~K}_{\mathrm{eq}}+c_{2} K_{2}-\frac{c_{1} c_{2}\left[\mathrm{~K}_{\mathrm{eq}}-K_{2}\right]^{2}}{c_{1} K_{2}+c_{2} \mathrm{~K}_{\mathrm{eq}}+4 / 3 \tilde{\mu}_{c}}$,

$\mu^{*}=c_{1} \mu_{\mathrm{eq}}+c_{2} \mu_{2}+\frac{4 c_{1} c_{2} \tilde{b}\left[\mu_{\mathrm{eq}}-\mu_{2}\right]^{2}}{1-4 \tilde{b}\left[c_{2} \mu_{\mathrm{eq}}+c_{1} \mu_{2}-\tilde{\mu}_{c}\right]}$,

where

$\tilde{b}=-\frac{3\left[\tilde{K}_{c}+2 \tilde{\mu}_{c}\right]}{10 \tilde{\mu}_{c}\left[3 \tilde{K}_{c}+4 \tilde{\mu}_{c}\right]}$

and $\tilde{K}_{c}, \tilde{\mu}_{c}$ are follows:

$\tilde{K}_{c}=\left\{\begin{array}{ll}c_{1} \mathrm{~K}_{\mathrm{eq}}+c_{2} K_{2}, & \text { if } \mathrm{K}_{\mathrm{eq}} \leq K_{2} \\ {\left[c_{1}\left(\mathrm{~K}_{\mathrm{eq}}\right)^{-1}+c_{2}\left(K_{2}\right)^{-1}\right]^{-1},} & \text { if } \mathrm{K}_{\mathrm{eq}} \geq K_{2}\end{array}\right.$,

$\tilde{\mu}_{c}= \begin{cases}c_{1} \mu_{\mathrm{eq}}+c_{2} \mu_{2}, & \text { if } \mu_{\mathrm{eq}} \leq \mu_{2} \\ {\left[c_{1}\left(\mu_{\mathrm{eq}}\right)^{-1}+c_{2}\left(\mu_{2}\right)^{-1}\right]^{-1},} & \text { if } \mu_{\mathrm{eq}} \geq \mu_{2}\end{cases}$

In the above formulas $K_{2}$ and $\mu_{2}$ are the bulk and shear moduli of the matrix, whereas $\mathrm{K}_{\mathrm{eq}}$ and $\mu_{\mathrm{eq}}$ are determined in Eqs. (50), (51), (26).

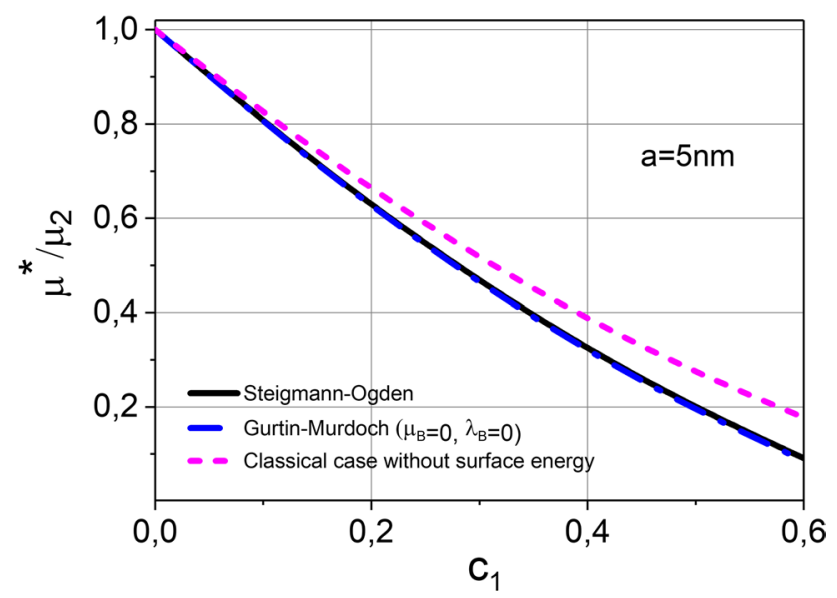

Fig. 1 Dependence of shear modulus $\mu^{*} / \mu_{2}$ for nanoporous silver on void volume fraction $c_{1}$; radius of a spherical cavity $a=5(\mathrm{~nm})$

\section{Numerical comparisons and discussion}

As a numerical example, we consider a composite material consisting of silver matrix with the properties $E_{2}=50 \mathrm{GPa}$ and $v_{2}=0.37$ containing spherical cavities $\left(\mu_{1}=K_{1}=0\right)$. The free surface properties are those presented by Mohammadi and Sharma [22]. In their article surface properties are determined for surface [100]: $\tau_{0}=0.3701 \mathrm{~N} / \mathrm{m}$; $\mu_{S}=-2.6948 \mathrm{~N} / \mathrm{m} ; \mu_{B}=12.3 \cdot 10^{-19} \mathrm{Nm}$. In the article, results are presented only for shear moduli. Assuming that relationship between Lamé parameters $\lambda$ and $\mu$ for bulk material and Lamé parameters for surface tension and for surface bending are the same we added missing parameters using relationship between Lamé parameters for bulk silver $\lambda / \mu=2.85$. So, in our calculation we take hypothetical values of $\lambda_{S}=-7.69 \mathrm{~N} / \mathrm{m} ; \lambda_{B}=35 \cdot 10^{-19} \mathrm{~N} \cdot \mathrm{m}$.

The variation of the normalized shear modulus $\mu^{*} / \mu_{2}$ with the void volume fractions calculated by the MCM in combination with EEI approach for the spherical cavities of radius $a=5 \mathrm{~nm}$ with Steigmann-Ogden model of interface is shown in Fig. 1 (black solid line). For comparison, the normalized shear modulus for the same material obtained accounting only for parameters of Gurtin-Murdoch interface model $\left(\mu_{B}=0, \lambda_{B}=0\right)$ (dash dot line) and for classical case without surface energy (short dash line) are also shown in Fig. 1. It is seen that difference between the normalized shear moduli obtained with accounting for SteigmannOgden and for Gurtin-Murdoch models of interface (when bending surface parameters are neglected) differ insignificantly what is illustrated in Fig. 2, which is a zoomed section of Fig. 1. It means that for case of interface whose thickness is vanishingly small, the Gurtin-Murdoch model of interface is dominant and influence of the surface bending on

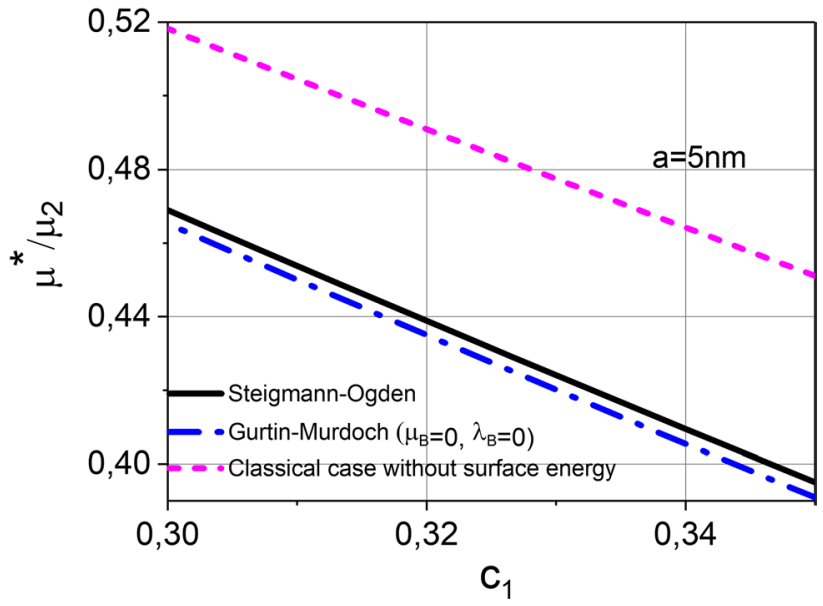

Fig. 2 Dependence of shear modulus $\mu^{*} / \mu_{2}$ for nanoporous silver on void volume fraction $c_{1}$; radius of a spherical cavity $a=5(\mathrm{~nm})$ (zoomed section of Fig. 1) 
effective properties is negligibly small. At the same time, the normalized shear moduli obtained with accounting only for Gurtin-Murdoch and for classical case without surface energy differ essentially, especially for high pore volume fraction. As expected, this numerical illustration indicates that the influence of the additional surface bending parameters of the Steigmann-Ogden model is insignificant for the shear moduli. It can be explained by the fact, that as shown by Benveniste and Miloh [1] the problem of an inhomogeneity with a thin interphase layer can be reduced to that of an imperfectly bonded inhomogeneity with the interface conditions described by one of seven distinct regimes, which depend on the elastic properties of the interphase layer and the components of composite. The Gurtin-Murdoch model corresponds to the membrane type interface regime, while the Steigmann-Ogden model corresponds to the inextensible shell regime. The surface bending stiffness of the membrane type interface is negligible, and the results presented in the manuscript support this. However, there may be a special case where the bending stiffness of the surface should be taken into account (e.g. [46].

As a second numerical result, we consider porous material with spherical cavities of radius $a=5 \mathrm{~nm}$ and following parameters adopted after Zemlyanova and Mogilevskaya [35]: $v_{2}=0.3, \tau_{0}=0 \mathrm{~N} / \mathrm{m} ; \frac{\mu_{S}}{\mu_{2} a}=0.030156 ; \frac{\lambda_{S}}{\mu_{2} a}=0.060312$; $\frac{5 \mu_{B}+3 \lambda_{B}}{\mu_{2} a^{3}}=0.00028382$.

The variation of the normalized shear modulus $\mu^{*} / \mu_{2}$ with void volume fractions calculated by the MCM in combination with the EEI approach (MCM EEI black solid line) for Steigmann-Ogden model of interface and for classical case without surface energy (MCM dash dot line) is shown in Fig. 3. For comparison analogical results for the normalized effective shear modulus with Steigmann-Ogden model

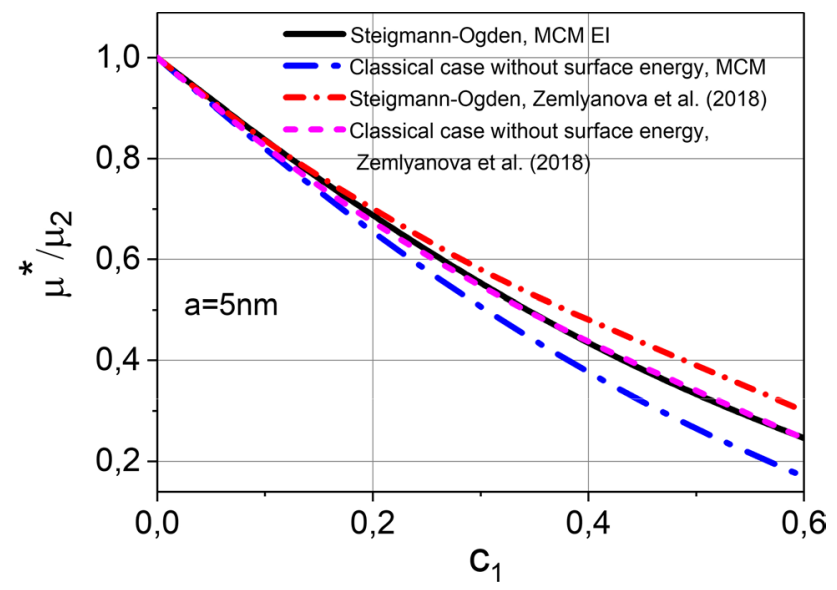

Fig. 3 Dependence of shear modulus $\mu^{*} / \mu_{2}$ for nanoporous material on void volume fraction $c_{1}$ (MCM EI and Zemlyanova et al. [35]); radius of a spherical cavity $a=5(\mathrm{~nm})$

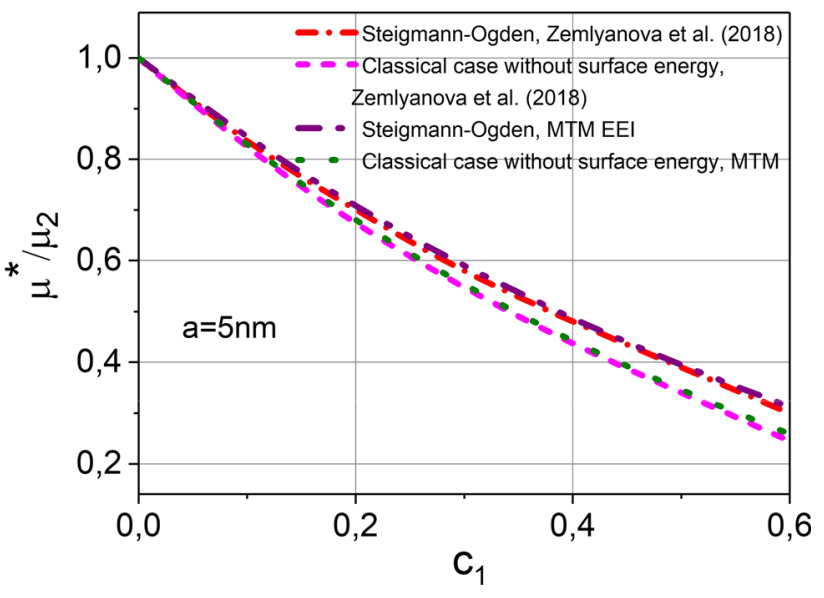

Fig. 4 Dependence of shear modulus $\mu^{*} / \mu_{2}$ for nanoporous material on void volume fraction $c_{1} c_{1}$ (MTM EI and Zemlyanova et al. [35]); radius of a spherical cavity $a=5(\mathrm{~nm})$

of interface and for the classical case obtained in Zemlyanova and Mogilevskaya [32, 35] (short dash dot line and short dash line) are presented in Fig. 3 as well. The results of Zemlyanova and Mogilevskaya [35] were obtained: for equivalent inhomogeneity on the base of exact Lurie's solution [40] for spherical particle and for effective properties of entire composite on the base of Maxwell homogenization scheme [49]. As it is seen, tendencies and numerical values for the results calculated by different methods demonstrate that the effective shear modulus of considered porous material presented in Zemlyanova and Mogilevskaya [35] are higher than those determined by EEI approach in combination with MCM. It can be explained by the conceptual differences in the MCM and Maxwell scheme, which in the case of material with spherical cavities is identical to Mori-Tanaka method (MTM) [48]. It is known, that for porous material MTM shows upper bound for effective moduli. For confirmation of this explanation the normalized effective shear modulus with Steigmann-Ogden model of interface and for the classical case were calculated by EEI approach in combination with MTM (MTM EEI short dash dot line and MTM dot line). These results and those of Zemlyanova and Mogilevskaya [35] are shown in Fig. 4. It is seen that the shear moduli calculated by MTM in combination with EEI approach are identical with the both obtained on the base of Maxwell scheme in combination with exact Lurie's solution. It means, that the contribution of Steigmann-Ogden interface to effective properties of entire composite is identical for the both cases. It can be considered as additional verification of accuracy of EEI approach where properties of equivalent inhomogeneity are identical with those obtained on the base of Lurie's solution. This is a very positive sign for the present approach given that in the definition of equivalent inhomogeneity based on Lurie's solution all governing equations 
in the inhomogeneity/interphase system are satisfied exactly. At the same time, the effective properties of entire composite calculated by MCM are lower than upper bound calculated by MTM.

Remark It should be noted that properties of equivalent spherical inhomogeneity with spring layer interphase obtained by EEI approach [38] have been compared with those calculated using the equivalent inhomogeneity defined on the base of Lurie's solution [38]. It was observed, that both solutions are virtually identical for all volume fraction of inhomogeneities.

\section{Conclusions}

A mathematical model, employing the concept of the EEI in combination with the MCM $[37,39,50]$, has been generalized to introduce the surface effects described by the Steigmann-Ogden model $[19,20]$ derived within the strain gradient elasticity [31]. A particular focus was centered on accounting for the surface bending contribution in the definition of the EEI. The model was also used in combination with MCM to determine the effective properties of materials with randomly distributed nano-particles with SteigmannOgden model of interface. Effective shear modulus of spherical EEI with Steigmann-Ogden model of interface is presented in a closed-form and compared with those obtained on the base of Lurie's solution for sphere.

The properties of the EEI are determined based on the derived definition of surface energy, which includes the surface tension and the surface bending, assuming the uniform state of strains within the inhomogeneity. This assumption is particularly suitable in the context of the MCM used here. The restriction of the MCM to the two-point approximation adopted here is tantamount to the assumption that deformation of the inhomogeneities is uniform. This is in perfect agreement with the way the EEI is defined in Sect. 3.

As a numerical illustration of the presented approach, nanoporous silver is studied. It is shown that the equivalent bulk modulus of spherical equivalent inhomogeneity does not depend on the surface bending parameters.

Shear moduli of nanoporous silver has been analyzed for varying volume content of the nano-cavities for SteigmannOgden model of interface and for classical case without accounting for the surface effects. The size effect introduced due to contribution of the residual stresses, elasticity and bending of the matrix/nanoparticles interface in nanoporous silver is accounted for in the expressions for effective bulk and shear moduli of the composite. It has been shown that the contribution of the surface bending to the shear moduli is insignificant.
As a second numerical example, the effective shear moduli of the porous material, evaluated on the basis of MCM in combination with EEI approach was considered in context of comparison with numerical results available in the literature and, is in a good agreement with one calculated in Zemlyanova and Mogilevskaya [32, 35]. It is interesting to note that, in spite of the difference between expressions for the shear modulus of equivalent inhomogeneity based on EEI and that obtained on the base of Lurie's solution for sphere, the effective properties of the composite calculated using those two definitions of the equivalent inhomogeneity are virtually identical for all volume fraction of voids. This indicates that the basic assumptions underlying the proposed approach are quite sound, as the approach based on Lurie's solution exactly fulfills all governing equations within the original inhomogeneity and the interphase.

To conclude it is worth mentioning that the definition of the EEI is general and can be used in the case of inhomogeneities of other shapes than spherical, e.g., ellipsoidal or cylindrical (e.g. [36]. It can be very naturally combined with the MCM and appears to be potentially amenable for inclusion of other than Gurtin-Murdoch or Steigmann-Ogden interface models. Therefore, the MCM with combination of the EEI can be applied for analysis of materials containing inhomogeneities with more complex geometric and mechanical characteristics. The important characteristic of the proposed approach is its ability to provide closed-form expressions for the effective properties of nano-composites. Closed-form results are important, especially if the influence of different problem parameters needs to be analyzed.

\section{Appendix 1: Components of curvature tensors for sphere}

Let's assume that the surface of interest is locally parameterized by $\xi^{\Lambda}, \Lambda \in\{1,2\}$, that is the position vector of a point on that surface is expressed as $\mathbf{r}\left(\xi^{\Lambda}\right)$. Then, one can define a couple of vectors $\mathbf{G}_{\Lambda}$

$\mathbf{G}_{\Lambda}=\frac{\partial \mathbf{r}}{\partial \xi^{\Lambda}} \equiv \mathbf{r},_{\Lambda}$,

which forms the vector basis in the linear space tangent to the surface $S$, called the natural basis. Another basis in the same tangent space, denoted by $\mathbf{G}^{\Delta}$ and called dual or reciprocal, is defined via the following orthogonality condition

$\mathbf{G}_{\Lambda} \cdot \mathbf{G}^{\Delta}=\delta_{\Lambda}^{\Delta}$,

where the "." represents the "dot" (or "inner") product of vectors and $\delta_{\Lambda}^{\Delta}$ is the Kronecker "delta". The bases $\mathbf{G}_{\Lambda}$ and $\mathbf{G}^{\Delta}$ are functions of $\xi^{\Lambda}$ and their derivatives can be expressed by the well-known Gauss-Weingarten formulas (see Itskov 
[47], for example). For the natural basis these formulas are (cf. Equation (64) for notations)

$\mathbf{G}_{\Lambda}, \Sigma=\Gamma_{\Lambda \Sigma}^{\Delta} \mathbf{G}_{\Delta}+B_{\Lambda \Sigma} \mathbf{n} \equiv \Gamma_{\Lambda \Sigma}^{1} \mathbf{G}_{1}+\Gamma_{\Lambda \Sigma}^{2} \mathbf{G}_{2}+B_{\Lambda \Sigma} \mathbf{n}$,

with a unit vector $\mathbf{n}$ normal to the surface. Here (as shown in the above equation) an index repeated in the subscript and superscript position implies summation, $\Gamma_{\Lambda \Sigma}^{\Delta}=\mathbf{G}_{\Lambda, \Sigma} \cdot \mathbf{G}^{\Delta}$ are the components of the local curvature tensor are

$B_{\Lambda \Sigma}=\mathbf{G}_{\Lambda, \Sigma} \cdot \mathbf{n}$.

Equation (66) together with Eq. (64) imply that $B_{\Lambda \Sigma}=B_{\Sigma \Lambda}$ whereas definition of the Christoffel symbols and Eq. (64) imply the following symmetry property $\Gamma_{\Lambda \Sigma}^{\Omega}=\Gamma_{\Sigma \Lambda}^{\Omega}$. The analogical formulas for the derivatives of vectors of the dual basis are

where $B_{\Sigma}^{\Delta}$ are the so-called mixed components of the local curvature tensor.

The curvature tensor $\mathbf{B}$ (components of which appear in Eqs. (66) and (67)) can be represented in several ways shown below (as well as many other ways)

$\mathbf{B}=B_{\Delta \Lambda} \mathbf{G}^{\Delta} \otimes \mathbf{G}^{\Lambda}=B^{\Delta \Lambda} \mathbf{G}_{\Delta} \otimes \mathbf{G}_{\Lambda}=B_{\Delta}^{\Lambda} \mathbf{G}^{\Delta} \otimes \mathbf{G}_{\Lambda}=B_{\Lambda}^{\Delta} \mathbf{G}_{\Delta} \otimes \mathbf{G}^{\Lambda}$ the so-called Christoffel symbols (of the second kind) and

$\mathbf{G}^{\Delta},_{\Sigma}=-\Gamma_{\Lambda \Sigma}^{\Delta} \mathbf{G}^{\Lambda}-B_{\Sigma}^{\Delta} \mathbf{n} \equiv-\Gamma_{1 \Sigma}^{\Delta} \mathbf{G}^{1}-\Gamma_{2 \Sigma}^{\Delta} \mathbf{G}^{2}-B_{\Sigma}^{\Delta} \mathbf{n}$,

$$
\mathbf{r}=a\left[\begin{array}{c}
\cos \phi \sin \theta \\
\sin \phi \sin \theta \\
-\cos \theta
\end{array}\right], \mathbf{n}=\left[\begin{array}{c}
\cos \phi \sin \theta \\
\sin \phi \sin \theta \\
-\cos \theta
\end{array}\right], 0 \leq \varphi \leq 2 \pi, 0 \leq \theta \leq \pi
$$

The natural basis is

$$
\begin{aligned}
& \mathbf{G}_{1}=r_{, \phi}=a\left[\begin{array}{c}
-\sin \theta \sin \phi \\
\sin \theta \cos \phi \\
0
\end{array}\right], \\
& \mathbf{G}_{2}=r_{, \theta}=a\left[\begin{array}{c}
\cos \phi \cos \theta \\
\sin \phi \cos \theta \\
\sin \theta
\end{array}\right], \\
& \mathbf{G}_{3}=\mathbf{G}^{3}=\mathbf{n}=\left[\begin{array}{c}
\cos \phi \sin \theta \\
\sin \phi \sin \theta \\
-\cos \theta
\end{array}\right],
\end{aligned}
$$

$\mathbf{G}_{1,1}=r_{, \phi \phi}=a\left[\begin{array}{c}-\sin \theta \cos \phi \\ -\sin \theta \sin \\ 0\end{array}\right]$

$\mathbf{G}_{2,1}=r_{, \theta \phi}=a\left[\begin{array}{c}-\sin \phi \cos \theta \\ \cos \phi \cos \theta \\ \sin \theta\end{array}\right]$

$\mathbf{G}_{2,2}=r_{, \theta \theta}=a\left[\begin{array}{c}-\cos \phi \sin \theta \\ -\sin \phi \sin \theta \\ \cos \theta\end{array}\right]$

The dual basis is defined as

$\mathbf{G}^{1}=\frac{1}{a}\left[\begin{array}{c}-\sin \phi / \sin \theta \\ \cos \phi / \sin \theta \\ 0\end{array}\right], \mathbf{G}^{2}=\frac{1}{a}\left[\begin{array}{c}\cos \phi \cos \theta \\ \sin \phi \cos \theta \\ \sin \theta\end{array}\right], \mathbf{G}_{3}=\mathbf{G}^{3}=\mathbf{n}=\left[\begin{array}{c}\cos \phi \sin \theta \\ \sin \phi \sin \theta \\ -\cos \theta\end{array}\right]$.
In the above equation double summation is implied and the (indexed) coefficients multiplying the dyadic are various components of the tensor B. They all can be different, but they are related to each other by transformation formulas involving the so-called Gram matrices related to the natural or dual bases. Those matrices are defined as follows

$G_{\Delta \Lambda}=\mathbf{G}_{\Delta} \cdot \mathbf{G}_{\Lambda}, G^{\Delta \Lambda}=\mathbf{G}^{\Delta} \cdot \mathbf{G}^{\Lambda}$.

The relationship between various components of the curvature tensor $\mathbf{B}$ one can present the following

$B_{\Delta}^{\Lambda}=B_{\Delta \Sigma} G^{\Sigma \Lambda}$.

In the case of spherical inhomogeneity of radius $a$, the position vector $\mathbf{r}$ of a point on the surface of the inhomogeneity, and the corresponding unit vector $\mathbf{n}$, normal to that surface, may be expressed as follows
Then the curvature tensors for spherical inhomogeneity of radius $a$ are given:

See Table 1

\section{Appendix 2: Properties of the energy-equivalent inhomogeneity accounting for surface bending}

For illustration of some technical details, $\mathbf{K}_{\mu_{B}}$ and $\mathbf{K}_{\lambda_{B}}$ of Eqs. (45)-(49) are evaluated in this Appendix. In addition to $\mathbf{K}_{\mu_{B}}$ and $\mathbf{K}_{\lambda_{B}}$, the contribution of surface tension to properties of equivalent inhomogeneity includes other term $\mathbf{K}_{T}$ present in Eq. (49), however evaluation of this term is presented in Nazarenko et al. [39]. 
Assuming that inhomogeneities are spheres of radius $a$ and using spherical coordinate system Eq. (72), $\mathbf{K}_{\mu_{B}}$ of Eqs. (45), (47), (48) is described by
$\hat{K}_{\mu_{B}}=K_{\mu_{B}[1111]}+K_{\mu_{B}[1122]}+K_{\mu_{B}[1133]}=4 \frac{\mu_{B}}{a^{3}}-2 \frac{\mu_{B}}{a^{3}}-2 \frac{\mu_{B}}{a^{3}}=0 ;$

$K_{\mu_{B}}=\frac{3 \mu_{B}}{2 \pi a^{3}} \int_{0}^{\pi} \int_{0}^{2 \pi}\left[\overline{\mathbf{G}}_{1} \otimes \overline{\mathbf{G}}_{1} \otimes \overline{\mathbf{G}}_{1} \otimes \overline{\mathbf{G}}_{1}+\overline{\mathbf{G}}_{2} \otimes \overline{\mathbf{G}}_{2} \otimes \overline{\mathbf{G}}_{2} \otimes \overline{\mathbf{G}}_{2}+2 \overline{\mathbf{G}}_{1} \otimes \overline{\mathbf{G}}_{2} \otimes \overline{\mathbf{G}}_{1} \otimes \overline{\mathbf{G}}_{2}-\overline{\mathbf{G}}_{1} \otimes \overline{\mathbf{G}}_{1} \otimes \mathbf{n} \otimes \mathbf{n}\right.$

$\left.-\overline{\mathbf{G}}_{2} \otimes \overline{\mathbf{G}}_{2} \otimes \mathbf{n} \otimes \mathbf{n}-\mathbf{n} \otimes \mathbf{n} \otimes \overline{\mathbf{G}}_{1} \otimes \overline{\mathbf{G}}_{1}-\mathbf{n} \otimes \mathbf{n} \otimes \overline{\mathbf{G}}_{2} \otimes \overline{\mathbf{G}}_{2}+2 \mathbf{n} \otimes \mathbf{n} \otimes \mathbf{n} \otimes \mathbf{n}\right] \sin \theta d \phi d \theta ;$
$K_{\mu_{b}[1111]}=\frac{3 \mu_{B}}{2 \pi r^{3}} \int_{0}^{\pi} \int_{0}^{2 \pi}\left[\sin \phi^{4}+\cos \phi^{4} \cos \theta^{4}\right.$

$+2 \sin \phi^{2} \cos \phi^{2} \cos \theta^{2}-2 \sin \phi^{2} \cos \phi^{2} \sin \theta^{2}$

$-2 \cos \phi^{4} \cos \theta^{2} \sin \theta^{2}$

$\left.+2 \cos \phi^{4} \sin \theta^{4}\right] \sin \theta d \phi d \theta=4 \frac{\mu_{B}}{a^{3}} ;$

$K_{\mu_{B}[2222]}=K_{\mu_{B}[3333]}=4 \frac{\mu_{B}}{\pi a^{3}}$

$K_{\mu_{B}[1122]}=\frac{3 \mu_{B}}{2 \pi a^{3}} \int_{0}^{\pi} \int_{0}^{2 \pi}\left[\sin \phi^{2} \cos \phi^{2}+\sin \phi^{2} \cos \phi^{2} \cos \theta^{4}-2 \sin \phi^{2} \cos \phi^{2} \cos \theta^{2}-\sin \phi^{4} \sin \theta^{2}\right.$

$\left.-\cos \phi^{4} \sin \theta^{2}-2 \sin \phi^{2} \cos \phi^{2} \cos \theta^{2} \sin \theta^{2}+2 \sin \phi^{2} \cos \phi^{2} \sin \theta^{4}\right] \sin \theta d \phi d \theta=-2 \frac{\mu_{B}}{a^{3}}$

$K_{\mu_{B}[1133]}=K_{\mu_{B}[2233]}=-2 \frac{\mu_{B}}{a^{3}}$

$K_{\mu_{B}[1212]}=\frac{3 \mu_{B}}{2 \pi a^{3}} \int_{0}^{\pi} \int_{0}^{2 \pi}\left[\sin \phi^{2} \cos \phi^{2}+\sin \phi^{2} \cos \phi^{2} \cos \theta^{4}+2 \sin \phi^{4} \cos \theta^{2}+2 \sin \phi^{2} \cos \phi^{2} \sin \theta^{2}\right.$

$\left.-2 \sin \phi^{2} \cos \phi^{2} \cos \theta^{2} \sin \theta^{2}+2 \sin \phi^{2} \cos \phi^{2} \sin \theta^{4}\right] \sin \theta d \phi d \theta=4 \frac{\mu_{B}}{a^{3}}$

$K_{\mu_{B}[1313]}=K_{\mu_{B}[2323]}=4 \frac{\mu_{B}}{a^{3}}$

$K_{\mu_{B}[2112]}=\frac{3 \mu_{B}}{2 \pi a^{3}} \int_{0}^{\pi} \int_{0}^{2 \pi}\left[\sin \phi^{2} \cos \phi^{2}+\sin \phi^{2} \cos \phi^{2} \cos \theta^{4}-2 \sin \phi^{2} \cos \phi^{2} \cos \theta^{2}+2 \sin \phi^{2} \cos \phi^{2} \sin \theta^{2}\right.$

$\left.-2 \sin \phi^{2} \cos \phi^{2} \cos \theta^{2} \sin \theta^{2}+2 \sin \phi^{2} \cos \phi^{2} \sin \theta^{4}\right] \sin \theta d \phi d \theta=2 \frac{\mu_{B}}{a^{3}}$

$K_{\mu_{B}[3113]}=K_{\mu_{B}[3223]}=2 \frac{\mu_{B}}{a^{3}}$.

Consequently, the contribution of $\mathbf{K}_{\mu_{B}}$ to bulk and shear moduli of the equivalent inhomogeneity are 


$$
\begin{aligned}
K_{\lambda_{B}}= & \frac{3 \lambda_{B}}{4 \pi a^{3}} \int_{0}^{\pi} \int_{0}^{2 \pi}\left[\overline{\mathbf{G}}_{1} \otimes \overline{\mathbf{G}}_{1} \otimes \overline{\mathbf{G}}_{1} \otimes \overline{\mathbf{G}}_{1}\right. \\
& +\overline{\mathbf{G}}_{2} \otimes \overline{\mathbf{G}}_{2} \otimes \overline{\mathbf{G}}_{2} \otimes \overline{\mathbf{G}}_{2}+\overline{\mathbf{G}}_{1} \otimes \overline{\mathbf{G}}_{1} \otimes \overline{\mathbf{G}}_{2} \otimes \overline{\mathbf{G}}_{2} \\
& +\overline{\mathbf{G}}_{2} \otimes \overline{\mathbf{G}}_{2} \otimes \overline{\mathbf{G}}_{1} \otimes \overline{\mathbf{G}}_{1} \\
& -2 \overline{\mathbf{G}}_{1} \otimes \overline{\mathbf{G}}_{1} \otimes \mathbf{n} \otimes \mathbf{n}-2 \overline{\mathbf{G}}_{2} \otimes \overline{\mathbf{G}}_{2} \otimes \mathbf{n} \otimes \mathbf{n} \\
& -2 \mathbf{n} \otimes \mathbf{n} \otimes \overline{\mathbf{G}}_{1} \otimes \overline{\mathbf{G}}_{1}-2 \mathbf{n} \otimes \mathbf{n} \otimes \overline{\mathbf{G}}_{2} \otimes \overline{\mathbf{G}}_{2} \\
& +4 \mathbf{n} \otimes \mathbf{n} \otimes \mathbf{n} \otimes \mathbf{n}] \sin \theta d \phi d \theta
\end{aligned}
$$

$$
K_{\lambda_{B}[3113]}=K_{\lambda_{B}[3223]}=\frac{9}{5} \frac{\lambda_{B}}{a^{3}} .
$$

The contribution of $\mathbf{K}_{\lambda_{B}}$ to bulk and shear moduli of the equivalent inhomogeneity are

$$
\begin{aligned}
& \hat{K}_{\lambda_{B}}=K_{\left.\lambda_{B}[111]\right]}+K_{\lambda_{B}[1122]}+K_{\lambda_{B}[1133]}=\frac{12}{5} \frac{\lambda_{B}}{a^{3}}-\frac{6}{5} \frac{\lambda_{B}}{a^{3}}-\frac{6}{5} \frac{\lambda_{B}}{a^{3}}=0 ; \\
& \hat{\mu}_{B}=\frac{1}{2}\left[K_{\lambda_{B}[1212]}+K_{\lambda_{B}[2112]}\right]=\frac{9}{5} \frac{\lambda_{B}}{a^{3}} .
\end{aligned}
$$

$$
\begin{aligned}
K_{\lambda_{B}[1111]}= & \frac{3 \lambda_{B}}{4 \pi a^{3}} \int_{0}^{\pi} \int_{0}^{2 \pi}\left[\sin \phi^{4}+\cos \phi^{4} \cos \theta^{4}+2 \sin \phi^{2} \cos \phi^{2} \cos \theta^{2}-4 \sin \phi^{2} \cos \phi^{2} \sin \theta^{2}\right. \\
& \left.-4 \cos \phi^{4} \cos \theta^{2} \sin \theta^{2}+4 \cos \phi^{4} \sin \theta^{4}\right] \sin \theta d \phi d \theta=\frac{12}{5} \frac{\lambda_{B}}{a^{3}}
\end{aligned}
$$

$K_{\lambda_{B}[2222]}=K_{\lambda_{B}[3333]}=\frac{12}{5} \frac{\lambda_{B}}{a^{3}} ;$

The contribution of surface bending to bulk and shear moduli of the equivalent inhomogeneity Eq. (28) is

$$
\begin{aligned}
K_{\lambda_{B}[1122]} & =\frac{3 \lambda_{B}}{4 \pi a^{3}} \int_{0}^{\pi} \int_{0}^{2 \pi}\left[\sin \phi^{2} \cos \phi^{2}+\sin \phi^{2} \cos \phi^{2} \cos \theta^{4}+\sin \phi^{4} \cos \theta^{2}\right. \\
& +\cos \phi^{4} \cos \theta^{2}-2 \sin \phi^{4} \sin \theta^{2}-2 \cos \phi^{4} \sin \theta^{2} \\
& \left.-4 \cos \phi^{2} \sin \phi^{2} \cos \theta^{2} \sin \theta^{2}+4 \cos \phi^{2} \sin \phi^{2} \sin \theta^{4}\right] \sin \theta d \phi d \theta=-\frac{6}{5} \frac{\lambda_{B}}{a^{3}}
\end{aligned}
$$

$$
K_{\lambda_{B}[1133]}=K_{\lambda_{B}[2233]}=-\frac{6}{5} \frac{\lambda_{B}}{a^{3}}
$$

$$
\hat{K}_{B}=\hat{K}_{B}+\hat{K}_{B}=0
$$

$$
\begin{aligned}
K_{\lambda_{b}[1212]} & =\frac{3 \lambda_{b}}{4 \pi r^{3}} \int_{0}^{\pi} \int_{0}^{2 \pi}\left[\sin \phi^{2} \cos \phi^{2}+\sin \phi^{2} \cos \phi^{2} \cos \theta^{4}\right. \\
& -2 \sin \phi^{2} \cos \phi^{2} \cos \theta^{2}+4 \sin \phi^{2} \cos \phi^{2} \sin \theta^{2} \\
& \left.-4 \cos \phi^{2} \sin \phi^{2} \cos \theta^{2} \sin \theta^{2}+4 \cos \phi^{2} \sin \phi^{2} \sin \theta^{4}\right] \sin \theta d \phi d \theta=\frac{9}{5} \frac{\lambda_{B}}{a^{3}}
\end{aligned}
$$

$$
K_{\lambda_{B}[1313]}=K_{\lambda_{B}[2323]}=\frac{9}{5} \frac{\lambda_{B}}{a^{3}} ;
$$

$$
\begin{aligned}
K_{\lambda_{B}[2112]} & =\frac{3 \lambda_{B}}{4 \pi a^{3}} \int_{0}^{\pi} \int_{0}^{2 \pi}\left[\sin \phi^{2} \cos \phi^{2}+\sin \phi^{2} \cos \phi^{2} \cos \theta^{4}\right. \\
& -2 \sin \phi^{2} \cos \phi^{2} \cos \theta^{2}+4 \sin \phi^{2} \cos \phi^{2} \sin \theta^{2} \\
& \left.-4 \cos \phi^{2} \sin \phi^{2} \cos \theta^{2} \sin \theta^{2}+4 \cos \phi^{2} \sin \phi^{2} \sin \theta^{4}\right] \sin \theta d \phi d \theta=\frac{9}{5} \frac{\lambda_{B}}{a^{3}}
\end{aligned}
$$


$\hat{\mu}_{B}=\hat{\mu}_{B}+\hat{\mu}_{B}=3 \frac{\mu_{B}}{a^{3}}+\frac{9 \lambda_{B}}{5 a^{3}}=\frac{15 \mu_{B}+9 \lambda_{B}}{5 a^{3}}$.

Acknowledgements LN and HA gratefully acknowledge the financial support by the German Research Foundation (DFG) via Project AL 341/51-1.

Funding Open Access funding enabled and organized by Projekt DEAL.

Open Access This article is licensed under a Creative Commons Attribution 4.0 International License, which permits use, sharing, adaptation, distribution and reproduction in any medium or format, as long as you give appropriate credit to the original author(s) and the source, provide a link to the Creative Commons licence, and indicate if changes were made. The images or other third party material in this article are included in the article's Creative Commons licence, unless indicated otherwise in a credit line to the material. If material is not included in the article's Creative Commons licence and your intended use is not permitted by statutory regulation or exceeds the permitted use, you will need to obtain permission directly from the copyright holder. To view a copy of this licence, visit http://creativecommons.org/licenses/by/4.0/.

\section{References}

1. Benveniste Y, Miloh T (2001) Imperfect soft and stiff interfaces in two-dimensional elasticity. Mech Mater 33:309-323

2. Dong CY, Zhang GL (2013) Boundary element analysis of three dimensional nanoscale inhomogeneities. Int J Solids Struct 50:201-208

3. Dong H, Wang J, Rubin M (2014) Cosserat interphase models for elasticity with application to the interphase bonding a spherical inclusion to an infinite matrix. Int J Solid Struct 51(2):462-477

4. Hashin Z (1990) Thermoelastic properties of fiber composites with imperfect interface. Mech Mater 8:333-348

5. Hashin Z (1991) Thermoelastic properties of particulate composites with imperfect in-terface. J Mech Phys Solids 39:745-762

6. He LH, Li ZR (2006) Impact of surface stress on stress concentration. Int J Solids Struct 43:6208-6219

7. Huang ZP, Wang J (2006) A theory of hyperelasticity of multi-phase media with surface/interface effect. Acta Mech 182:195-210

8. Mi C, Kouris DA (2006) Nanoparticles under the influence of surface/interface elasticity. Mech Mater Struct 1:763-791

9. Rubin M, Benveniste Y (2014) A Cosserat shell model for interphases in elastic media. J Mech Phys Solid 52(5):1023-1052

10. Zhang WX, Wang T (2007) Effect of surface energy on the yield strength of nanoporous materials. Appl Phys Lett 90, Art. No. 063104

11. Gurtin ME, Murdoch AI (1975) A continuum theory of elastic material surfaces. Arch Ration Mech Anal 57(4):291-323

12. Gurtin ME, Murdoch AI (1978) Surface stress in solids. Int J Solids Struct 14:431-440

13. Brisard S, Dormieux L, Kondo D (2010) Hashin-Shtrikman bounds on the bulk modulus of a nanocomposite with spherical inhomogeneities and interface effects. Comput Mater Sci 48(3):589-596

14. Chen T, Dvorak GJ, Yu CC (2007) Size-dependent elastic properties of unidirectional nano-composites with interface stresses. Acta Mech 188:39-54

15. Duan HL, Wang J, Huang ZP, Karihaloo BL (2005) Sizedependent effective elastic constants of solids containing nanoinhomogeneities with interface stress. J Mech Phys Solids 53:1574-1596

16. Lim CW, Li ZR, He LH (2006) Size-dependent, non-uniform elastic field inside a nano-scale spherical inclusion due to interface stress. Int J Solids and Struct 43:5055-5065

17. Miller RE, Shenoy VB (2000) Size-dependent elastic properties of nanosized structural elements. Nanotechnology 11:139-147

18. Altenbach H, Eremeyev VA (2011) On the shell theory on the nanoscale with surface stresses. Int J Eng Sci 49:1294-1301

19. Steigmann DJ, Ogden RW (1997) Plane deformations of elastic solids with intrinsic boundary elasticity. Proc R Soc Lond A 453:853-877

20. Steigmann DJ, Ogden RW (1999) Elastic surface-substrate interactions. Proc R Soc Lond A 455:437-474

21. Chhapadia P, Mohammadi P, Sharma P (2011) Curvature-dependent surface energy and implications for nanostructures. J Mech Phys Solids 59:2103-2115

22. Mohammadi P, Sharma P (2012) Atomistic elucidation of surface roughness on curvature-dependent surface energy, surface stress, and elasticity. Appl Phys Latter 100:133110-1-133114

23. Javili A, Dell'Isola F, Steinmann P (2013) Geometrically nonlinear higher-gradient elasticity with energetic boundaries. J Mech Phys Solids 61:2381-2401

24. Javili A, Ottosen NS, Ristinmaa M, Mosler J (2018) Aspects of interface elasticity theory. Math Mech of Solids 23(7):1004-1024

25. Dell'Isola F, Seppecher P (1997) Edge contact forces and quasibalanced power. Meccanica 32(1):33-52

26. Dell'Isola F, Seppecher P, Madeo A (2012) How contact interactions may depend on the shape of Cauchy cuts in Nth gradient continua: approach "à la d'Alembert." Z Angew Math Phys 63:1119-1141

27. Mindlin RD (1964) Micro-structure in linear elasticity. Arch Ration Mech Anal 16(1):51-78

28. Mindlin RD (1965) Second gradient of strain and surface-tension in linear elasticity. Int J Solids Struct 1(4):417-438

29. Toupin RA (1962) Elastic materials with couple-stresses. Arch Ration Mech Anal 11(1):385-414

30. Eremeyev VA, Lebedev LP (2016) Mathematical study of boundary-value problems within the framework of Steigmann-Ogden model of surface elasticity. Continuum Mech Therm 28:407-422

31. Eremeev V (2019) On dynamic boundary conditions within the linear Steigmann-Ogden model of surface elasticity and strain gradient elasticity. In: Altenbach H, Belyaev A, Eremeyev V, Krivtsov A, Porubov A (eds) Dynamical processes in generalized continua and structures advanced structured materials, Vol 103. Cham, Springer

32. Zemlyanova AY, Mogilevskaya SG (2018a) Circular inhomogeneity with Steigmann-Ogden interface: local fields, neutrality, and Maxwell's type approximation formula. Int J Solids Struct 135:85-98

33. Gao X, Huang Z, Qu J, Fang D (2014) A curvature-dependent interfacial energy-based interface stress theory and its applications to nanostructured materials: (I) general theory. J Mech Phys Solids 66:59-77

34. Gao X, Huang Z, Fang D (2017) Curvature-dependent interfacial energy and its effects on the elastic properties of nanomaterials. Int J Solid Struct 113:100-107

35. Zemlyanova AY, Mogilevskaya SG (2018b) On spherical inhomogeneity with Steigmann-Ogden interface. J Appl Mech 85(12): 121009

36. Nazarenko L, Stolarski H, Altenbach H (2020) Modeling cylindrical inhomogeneity of finite length with steigmann-ogden interface. Technologies 8:78. https://doi.org/10.3390/technologies804 0078

37. Nazarenko L, Bargmann S, Stolarski H (2015) Energy-equivalent inhomogeneity approach to analysis of effective properties 
of nano-materials with stochastic structure. Int J Solids Struct 59:183-197

38. Nazarenko L, Stolarski H (2016) Energy-based definition of equivalent inhomogeneity for various interphase models and analysis of effective properties of particulate composites. Comp Part B 94:82-94

39. Nazarenko L, Bargmann S, Stolarski H (2017) Closed-form formulas for the effective properties of random particulate nanocomposites with complete Gurtin-Murdoch model of material surfaces. Continuum Mech Thermodyn 29:77-96

40. Lurie AI (2005) Theory of elasticity. Springer, Berlin Heidelberg

41. Hervé E, Zaoui A (1990) Modelling the effective behavior of nonlinear matrix-inclusion composites. J Eur J Mech A/Solids 9:505-515

42. Hervé E, Zaoui A (1993) n-Layered inclusion-based micromechanical modelling. Int J Eng Sci 31:1-10

43. Nazarenko L, Bargmann S, Stolarski H (2014) Influence of interfaces on effective properties of nanomaterials with stochastically distributed spherical inclusions. Int J Solids Struct 51:985-997

44. Nazarenko L, Khoroshun L, Müller WH, Wille R (2009) Effective thermoelastic properties of discrete-fiber reinforced materials with transversally-isotropic components. Cont Mech Thermodyn 20:429-458

45. Nazarenko L, Stolarski H, Altenbach H (2018) Effective thermoelastic properties of random composites with orthotropic components and aligned ellipsoidal inhomogeneities. Int J Solids Struct 136-137:220-240
46. Eremeyev VA, Wiczenbach T (2020) On effective bending stiffness of a laminate nanoplate considering Steigmann-Ogden surface elasticity. Appl Sci 10: 7402. https://doi.org/10.3390/app10 21740 .

47. Itskov $M$ (2007) Tensor algebra and tensor analysis for engineeers. Springer, Berlin

48. Mura T (1987) Micromechanics of defects in solids. Martinus Nijhoff Publishers, Dortrecht, The Netherlands

49. Sevostianov I, Giraud A (2013) Generalization of Maxwell homogenization scheme for elastic material containing inhomogeneities of diverse shape. Int J Eng Sci 64:23-36

50. Nazarenko L, Bargmann S, Stolarski H (2016) Lurie solution for spherical particle and spring layer model of interphases: its application in analysis of effective properties of composites. Mech Mater 96:39-52

51. Shenoy VB (2005) Atomistic calculations of elastic properties of metallic fcc crystal surfaces. Phys Rev B 71:094104

52. Stolarski HK, Belytschko T, Carpenter N (1983) Bending and shear mode decomposition in $\mathrm{C}^{\circ}$ structural elements. J Struct Mech 11(2):153-176

Publisher's Note Springer Nature remains neutral with regard to jurisdictional claims in published maps and institutional affiliations. 